\title{
Development of an Infrared Hollow Waveguide Sampler for the Detection of Organic Compounds in Aqueous Solutions with Limited Sample Volumes
}

\author{
Jyisy YANG*广 and Pon-Yen CHEN** \\ * Department of Chemistry, National Chung-Hsing University, Taichung, Taiwan 402 \\ **Department of Chemistry, Chung-Yuan Christian University, Chung-Li, Taiwan 320
}

\begin{abstract}
In this study, an infrared (IR) hollow waveguide sampler was developed to detect organic compounds in aqueous samples with sample volumes less than $50 \mu \mathrm{L}$. This sampler was prepared by coating a thin hydrophobic film inside the IR hollow waveguide. After injecting a certain amount of aqueous solution, organic compounds could be absorbed into the hydrophobic film by partitions. By removing the residual water in the hollow waveguide sampler with a nitrogen purging gas, the absorbed organic compounds could be sensed using IR radiation. To investigate the applicability of this hollow waveguide sampler in the detection of small amounts of aqueous samples, an analytical working function was developed following an examination of the parameters which influence the analytical signals. Such factors as the volume of the aqueous solution, the sample concentration, the length of the hollow waveguide, and the sensitivity of this method were investigated. Excellent agreement between the analytical and theoretical predicted values was observed. Upon examining the linear relationship between the analyte signals and the concentration, the regression coefficients were generally higher than 0.998 in the examined concentration range of 1 to $100 \mathrm{ppm}$. Under the condition that the sample volume was $300 \mu \mathrm{L}$ and based on three-times the spectra noise level, the calculated detection limits for this method were found at around $1 \mathrm{ppm}$ for the examined analytes.
\end{abstract}

(Received December 7, 2001; Accepted February 1, 2002)

\section{Introduction}

The size of a sample is one of the major considerations in selecting a suitable analytical method. In this study, we focused on the development of an infrared (IR) sensing method to extend the current required large sample volume limitation when Fourier transform infrared (FT-IR) spectrometry was applied to detect organic compounds in aqueous solutions. The results from the study will be beneficial to fields where limited sample volumes are the usual case, such as monitoring the reaction products in a reaction pot, examining biological samples and detecting analytes eluted from high performance liquid chromatography. Even when large aqueous sample amounts can be easily obtained, the cost reduction from reducing the need to handle large amounts of samples is worth developing methods that are effective with small samples.

During the past few years, FT-IR spectroscopic methods have been applied largely to detect organic compounds in aqueous solutions because of its speed and simplicity. Moreover, abundant chemical information can be obtained based on specific IR absorption bands. Because of the strong absorption of water molecules in aqueous solutions, the attenuated total reflection (ATR) ${ }^{1}$ effect has been used most frequently because of the short traveling distance of the evanescent wave limits absorption by the water molecules. Based on this effect, both un-coated ${ }^{2-6}$ and hydrophobic film coated ${ }^{7-17}$ internal reflection elements (IREs) have been used to detect organic compounds in

$\dagger$ To whom correspondence should be addressed.

E-mail: jyisy@dragon.nchu.edu.tw aqueous solutions. The sensitivity was greatly improved for methods using hydrophobic film-coated IRE. The coated hydrophobic film served as a solid phase micro-extraction phase (SPME) $)^{18-20}$ to exclude water molecules from the radiation penetration depth and to attract organic species to the sensing IREs. Although the present SPME/ATR-IR spectroscopic methods provide direct and convenient ways to detect organic compounds in aqueous solutions, the sensitivity is still limited because of the short evanescent wave penetration distance. In recent years, IR sensing methods based on an IR hollow waveguide have been proposed by our group. ${ }^{21-23}$ In this method, the inner surface of the IR waveguide is coated with a hydrophobic polymer, which serves as the SPME coating. When aqueous samples are passed through the tube, dissolved organic species can be absorbed into the SPME layer for further analysis using FT-IR spectrometry. Organic compounds with low volatility can be quantitatively detected using this method. Unlike ATR methods, in which the analytes must diffuse into the penetration depth, any organic species absorbed into the SPME layer of the hollow waveguide sampler can be detected by the reflection of the IR radiation inside the hollow waveguide. Compared to the ATR approach, this sampler type can also provide advantages, such as mass analyses, short analysis time and higher detection sensitivity. ${ }^{20-22}$ The large hydrophobic film surface inside the sampler can effectively contact with the aqueous solution to reduce the time to reach the equilibrium condition. Because of the advantages of a hollow waveguide sampler, it is worthwhile to extend this method to analyze aqueous samples with limited volumes. 


\section{Theoretical Aspects}

In the hollow waveguide sampler, organic-compound absorption is still based on the SPME principle. However, the SPME analysis working function should be corrected when only a limited sample amount is examined. Basically, the partition coefficient in a two-phase system is a concern with SPME, and can be expressed as the ratio of the solute concentration in the hydrophobic and aqueous phases, as shown by

$$
K=C_{\text {org }} / C_{\text {aq }},
$$

where $K$ is the partition coefficient, $C_{\text {org }}$ the analyte concentration in the hydrophobic layer and $C_{\mathrm{aq}}$ the solute concentration in the aqueous solution. This equation can be expressed in terms of the number of molecules absorbed after equilibrium is reached, as follows:

$$
\begin{aligned}
n & =\left(K \times n_{\mathrm{o}} \times V_{\mathrm{f}}\right) /\left(V_{\mathrm{s}}+V_{\mathrm{f}} \times K\right) \\
& =\left(K \times C_{\mathrm{o}} \times V_{\mathrm{s}} \times V_{\mathrm{f}}\right) /\left(V_{\mathrm{s}}+V_{\mathrm{f}} \times K\right),
\end{aligned}
$$

where $n$ is the number of molecules partitioned into the organic phase, $n_{\mathrm{o}}$ is the original number of molecules in the aqueous solution, $V_{\mathrm{f}}$ is the volume of the organic phase, and $V_{\mathrm{s}}$ is the volume of the aqueous solution. Under the condition that the sample volume is large enough, the above equation can be simplified in terms of the number of molecules absorbed by the SPME phase, as shown by

$$
n=K \times C_{\mathrm{o}} \times V_{\mathrm{f}}
$$

This equation indicates that the total amount of analyte that can be detected is proportional to the partition coefficient of the analytes, the volume of the SPME phase and the original concentration.

However, under the condition that the sample volume is not large enough to ignore, the contribution of $V_{\mathrm{f}} \times K$, Eq. (2) should be kept without simplification. To understand the relationship between the values of such factors as $V_{\mathrm{f}}, K$, and $V_{\mathrm{s}}$ and their contributions to the analytical signals, the SPME phase volume and the concentration of the analyte were first assumed to be constant and equal to $1 \mu \mathrm{L}$ and $10 \mathrm{ppm}$, respectively. The number of absorbed molecules can be calculated with respect to the sample volume $\left(V_{\mathrm{s}}\right)$ at different $K$ values. Using Eq. (3), the weight of the molecules absorbed from an extremely large sample volume can also be calculated. The calculated weights for absorbing $10 \mathrm{ppm}$ of organic aqueous sample were 10, 5, and $1 \mu \mathrm{g}$ for $K$ equal to 1000,500 and 100, respectively. The calculated weights of the absorbed organic molecules from different sample volumes are plotted in Fig. 1A. As can be seen in this plot, the sample volume size required for absorbing $50 \%$ of the molecules, as from an extremely large sample, can be lower than $1 \mathrm{~mL}$. For example, $1 \mu \mathrm{L}$ of hydrophobic film can absorb $1 \mu \mathrm{g}$ of the analytes from an excessively large sample volume when the analytes have a $K$ value of 100 . With a sample volume of $0.1 \mu \mathrm{L}, 0.5 \mu \mathrm{g}$ of the analytes can be absorbed into the hydrophobic film. When the analytes exhibited $K$ values of around 1000, the expected weight of the analytes absorbed into the hydrophobic film was $10 \mu \mathrm{g}$ with an excessively large sample volume. However, only $1 \mathrm{~mL}$ of the aqueous sample was required to absorb $5 \mu \mathrm{g}$ of the analytes.

Conversely, if the sample volume is limited, the maximum hydrophobic film sample can be optimized according to Eq. (2). Figure 1B shows the calculated number of molecules absorbed
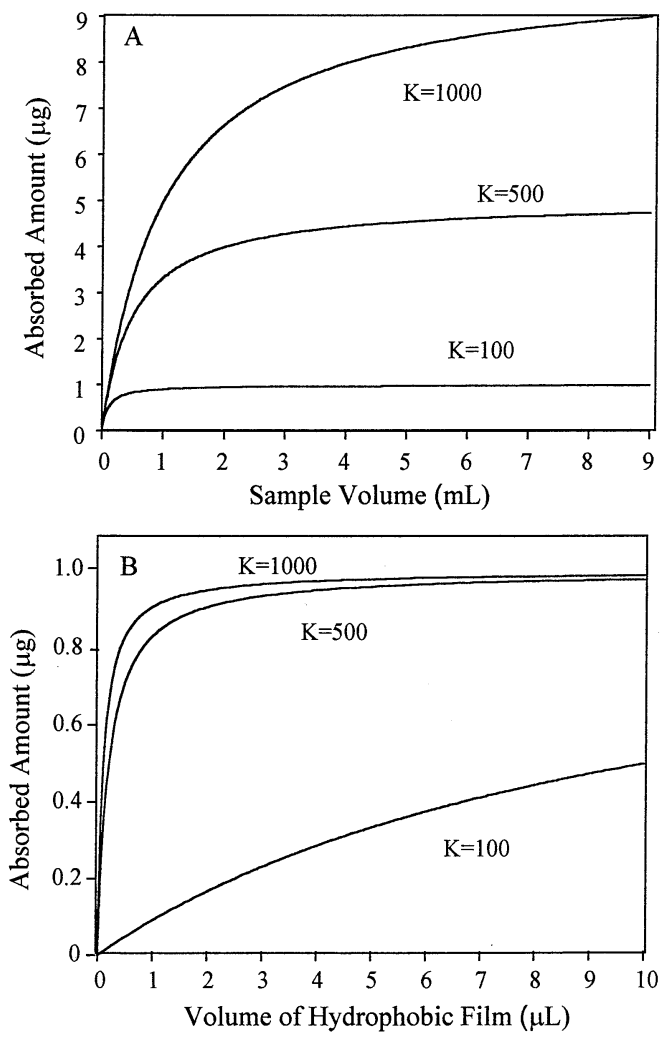

Fig. 1 (A) Theoretical calculation of the amount of analytes absorbed into hydrophobic film with respect to the volume of the sample. Three partition coefficients $(K)$ of analytes were calculated including 100,500 , and 1000 . The volume of hydrophobic film was assumed to be $1 \mu \mathrm{L}$, and the concentration of the sample was assumed to be $10 \mathrm{ppm}$. (B) Theoretical calculation of the amount of analytes absorbed into hydrophobic film with respect to the volume of the hydrophobic film. The volume of the sample was assumed to be $100 \mu \mathrm{L}$, and the concentration of the sample was assumed to be 10 ppm.

with different $V_{\mathrm{f}}$ under the condition that $V_{\mathrm{s}}$ is limited to $100 \mu \mathrm{L}$ and $K$ varies from 100, 500, and 1000 for $10 \mathrm{ppm}$ of the solution. As can be seen in this figure, for a large $K$ value, the required $V_{\mathrm{f}}$ is less. For example, when $V_{\mathrm{f}}$ is only $0.1 \mu \mathrm{L}, 50 \%$ of the molecules can be attracted for $K$ is equal to 1000 .

Generally speaking, to absorb $50 \%$ of the molecules into a hydrophobic film (compared to the excess sample volume), the value of $V_{\mathrm{s}}$ should be equal to $K \times V_{\mathrm{f}}$. Therefore, if $V_{\mathrm{f}}$ is fixed, the larger is the $K$ value, the larger is the required sample volume. Under the condition that the sample volume is limited, the minimal film thickness should be at least larger than the value of the value of $V_{\mathrm{s}} / K$. The larger is $K$, the smaller is the required $V_{\mathrm{f}}$. For organic compound partitioning in a two-phase (octane/water) system, the $K$ values should generally be in the range of 10 to $10^{5}$, and should be easily detected when a small volume is used. Although the percentage of analyte absorption is low for a large $K$ value, the actual amount of absorbed molecules with a low sample volume is still higher than that with a low $K$ value.

A quantitative analysis can also be performed when the sample amount is limited. If all of the parameters, such as $V_{\mathrm{s}}$, $V_{\mathrm{f}}$, and $K$, are kept constant, Eq. (2) can be expressed as

$$
n=K^{\prime} \times C_{\mathrm{o}},
$$



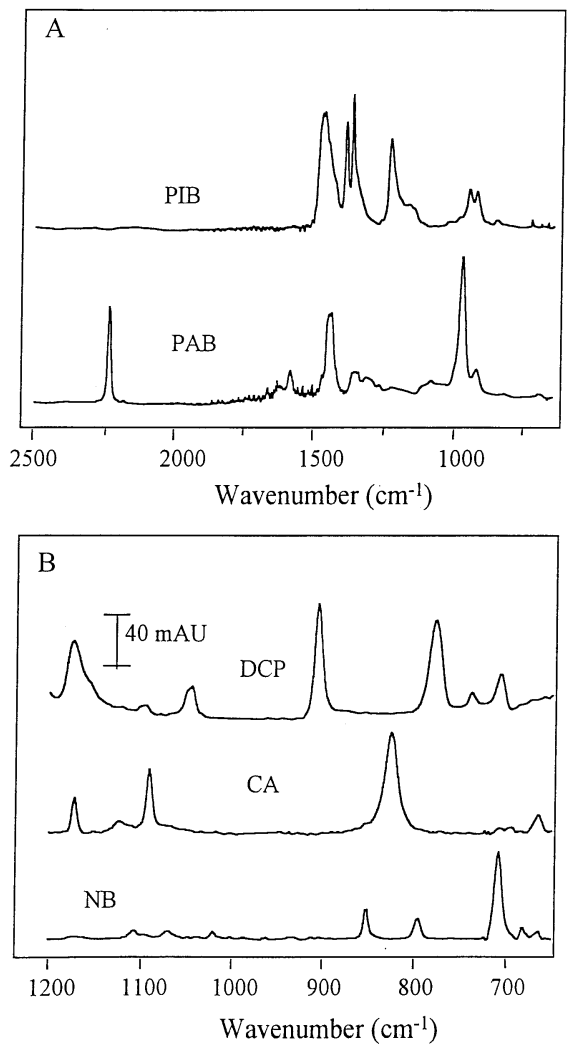

Fig. 2 (A) Typical spectra of hydrophobic films (PIB and PAB) used in this work. (B) Some typical spectra of 100-ppm 2,3-DCP, 4$\mathrm{CA}$, and NB obtained by the injection of $300 \mu \mathrm{L}$ of the sample into a $10-\mathrm{cm}$ hollow waveguide sampler for an equilibrium of $10 \mathrm{~min}$. The hydrophobic film was PAB in these experiments. The spectra were co-added with 100 scans in $4 \mathrm{~cm}^{-1}$ resolution.

where $K^{\prime}$ is equal to $\left(V_{\mathrm{s}} \times V_{\mathrm{f}}\right) /\left(V_{\mathrm{s}}+V_{\mathrm{f}} \times K\right)$, and should be constant if all of the parameters remain the same. Based on Eq. (4), the analytical signals should be linear with an increase in the concentration. Therefore, the quantitative results for aqueous samples with a limited amount of volume can be obtained.

\section{Experimental}

\section{Materials and reagents}

Polyethylene (PE) tubes obtained from a local supplier were used as the substrate for the producing a hollow waveguide. The internal and external diameters of the PE tube were $2.0 \mathrm{~mm}$ and $2.2 \mathrm{~mm}$, respectively. The PE tubes were cut into 1-meter lengths before chemical deposition on the inner surface. The chemicals used to form plating solutions were of reagent grade. These chemicals included silver nitrate and glucose (Prochem Inc., Rockford, IL), sulfuric acid, chromic acid, $\mathrm{SnCl}_{2}$, and $\mathrm{PdCl}_{2}$ (Acros Organics, New Jersey). Two polymeric materials, obtained from Aldrich (Milwaukee, WI), were used. These materials included polyisobutylne (PIB) and polyacrylonitrileco-butadiene (PAB). Because the chemical properties of PIB are similar to that of octane, the partition coefficients for the organic compounds in an octane/water system could be used to study the $K$ effect on the IR signals. The other polymer, PAB, has been studied ${ }^{9,22}$ concerning its properties to attract more polar organic compounds by our group. Previous results indicated that PAB absorbs more polar compounds effectively, especially for organic compounds with amine ${ }^{22}$ or hydroxyl groups. ${ }^{9}$ The monomer of acrylonitrile in $\mathrm{PAB}$ was in the range of $37 \%$ to $39 \%$ in mole ratio. Nine compounds obtained from Aldrich (Milwaukee, WI) were studied in this work, including 1-chloronaphthalene (1-CN), 2-chlorotoluene (2-CT), phenol, 4nitrophenol (4-NP), 4-chlorophenol (4-CP), 3-nitroaniline (3NA), 2,3-dichlorophenol (2,3-DCP), 2,4-dichloroaniline (2,4DCA), and acetophenone (AC). PIB was dissolved in toluene and used to coat the sampler for attracting 1-CN and 2-CT. $\mathrm{PAB}$ was dissolved in acetone and used to coat the sampler for attracting the remaining compounds. These solvents were obtained from TEDIA (Ohio, USA) in reagent grade.

\section{Production of a hollow IR waveguide sampler}

The production details for the hollow waveguide sampler are described in Ref. 21. Only a brief description of the hollow waveguide sampler is given here. The inner surface of a PE tube was first cleaned and treated with strong oxidants. After this process, a sensitization/activation process was followed using an $\mathrm{SnCl}_{2} / \mathrm{PdCl}_{2}$ solution. After rinsing with deionized water, a metallization operation was performed by separately pumping a silver solution and a reduction solution through the PE tube. The produced hollow waveguide possessed an infrared radiation transmission of around $15 \mathrm{~dB} / \mathrm{m}$. The waveguide was cut into $10-\mathrm{cm}$ long pieces and the inner surface was further coated with a polymer solution prepared in concentration ranges from 1 to $6 \%(\mathrm{w} / \mathrm{v})$. To coat the polymer film inside the hollow waveguide, a glass syringe was used to suck $3 \mathrm{~mL}$ of the polymer solution through the hollow waveguide. The coated hollow waveguides were air dried for $24 \mathrm{~h}$. Typical PIB and PAB spectra are shown in Fig. 2A. A PIB band located at 1230 $\mathrm{cm}^{-1}$ and an PAB band located at $2237 \mathrm{~cm}^{-1}$ were used to indicate the amount of polymer deposited inside the sampler. As can be seen in Fig. 2A, both polymers exhibit only a few absorption bands, which are present in the wavenumber region from 2500 to $650 \mathrm{~cm}^{-1}$. As described in the analytical function development section, the volume of the polymer film strongly influences the analytical signals. Therefore, a quantitative determination of the polymer film thickness (or volume) is necessary. Because the number of reflections inside the hollow waveguide was not known, the actual volume of the SPME phase could not be obtained precisely. Instead, an estimation method was used to roughly determine the thickness of the polymer film. Under conditions in which the molar absorption coefficient remained the same, the band intensities obtained from the PIB inside the sampler and from the PIB coated in the ZnSe window (known thickness and determined by placing a certain amount of PIB solution on the $\mathrm{ZnSe}$ surface) could be used to estimate the thickness of the polymer deposited inside the hollow waveguide. By assuming that the average number of reflections inside the hollow waveguide sampler was 20 and comparing the spectra of the deposited polymer film inside the sampler with the transmission spectra, the thickness of the deposited film was found to be around $0.3 \mu \mathrm{m}$ when the PIB absorption band was located at $1230 \mathrm{~cm}^{-1}$, showing an intensity of 0.716 absorbance units (AU).

\section{Procedure for sampling and detection}

Probe organic compounds were dissolved in water to form the desired concentrations. Samples were injected using a microsyringe to draw the desired amount of aqueous solution into a bent sampler. After equilibrium for a certain time, the aqueous solution was removed and regulated nitrogen gas was used to remove any residual water molecules. For absorbed analyte detection, two non-imaging infrared concentrators (modified 

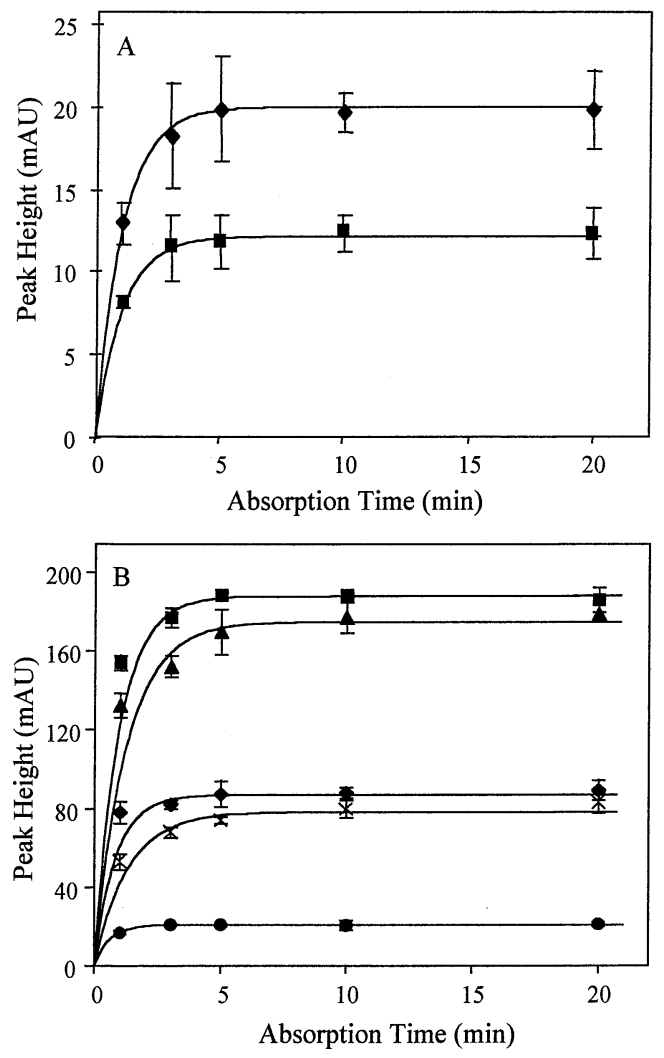

Fig. 3 (A) Typical absorption time profiles for 10-ppm 1-CN ( and 2-CT ( $)$ absorbed by PIB film. (B) Typical absorption time profiles for 100-ppm 4-NP ( $)$, 4-CP ( $)$, acetophenone ( $\bullet$ ), 2,3DCP $(x)$, and phenol $(\bullet)$ absorbed by PAB film. A 10-cm long sampler and $300 \mu \mathrm{L}$ samples were used in these experiments.

from Spectral-Tech fiber probe accessory) were placed at each end of the hollow waveguide sampler for concentrating the IR radiation from the sample compartment of an FT-IR spectrometer into the hollow waveguide and to redirect the transmitted IR radiation back into the MCT detector. A Nicolet Magna 550 FT-IR spectrometer equipped with a medium-range mercury-cadmium-telluride $\left(1 \mathrm{~mm}^{2}\right)$ detector was used to detect the absorbed analytes. The spectra presented in this work were coadded with 100 scans in $4 \mathrm{~cm}^{-1}$ resolution, unless specified. Some typical organic compound spectra detected using this method are shown in Fig. 2B. In this figure, $300 \mu \mathrm{L}$ of $100-\mathrm{ppm}$ organic compound was injected into the sampler after $10 \mathrm{~min}$ of equilibrium. This figure also shows that high quality IR spectra could be easily obtained. The amount of analytes absorbed by the polymer film was determined using the band intensities located at 766, 746, 757, 1338, 1495, 1528, 906, 1485, and 1685 $\mathrm{cm}^{-1}$, indicating the amount of analytes absorbed for 1-CN, 2CT, phenol, 4-NP, 4-CP, 3-NA, 2,3-DCP, 2,4-DCA, and AC, respectively.

\section{Results and Discussion}

Absorption time profiles for organic compounds detected in aqueous solutions with limited sample volumes

The equilibrium time is an important factor for obtaining optimal analytical signals without spending too much time. In order to study the efficiency in reaching the equilibrium conditions, the absorption time profiles of $1-\mathrm{CN}, 2-\mathrm{CT}$, phenol,
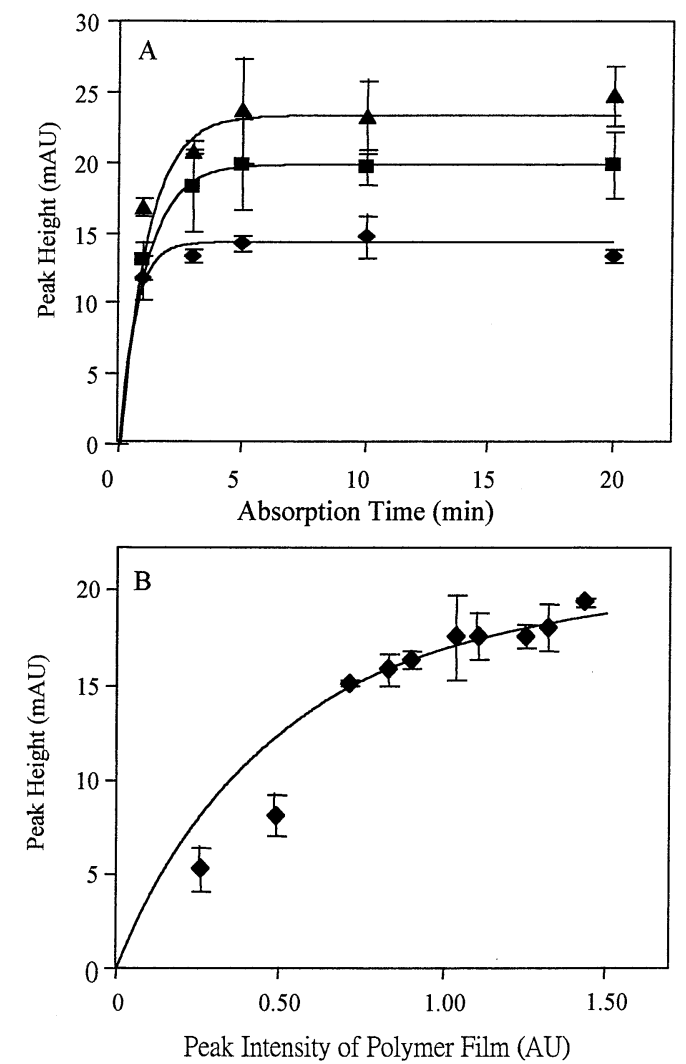

Fig. 4 (A) Typical absorption time profiles for the detection of 10ppm 1-CN using different thickness of PIB films. The thickness of PIB was determined by its absorption band intensity at $1230 \mathrm{~cm}^{-1}$ and the band intensities were $0.714(\bullet), 1.175(\boldsymbol{\square})$, and 1.516 AU ( $\mathbf{\Delta})$. (B) Relationship between the thickness of the PIB film and the analytical signals for 10-ppm 1-CN absorbed by PIB films. The equilibrium time was $20 \mathrm{~min}$ in this work. The thickness of PIB was plotted as the band intensity of PIB at $1230 \mathrm{~cm}^{-1}$.

4-NP, 4-CP, 2,3-DCP, and AC were studied. Because 1-CN and 2-CT exhibit lower polarity and can be effectively absorbed by PIB, these two compounds were dissolved in $10 \mathrm{ppm}$ and absorbed using the PIB phase. The remaining compounds contain a polar functional group, such as $-\mathrm{OH},-\mathrm{NO}_{2},-\mathrm{C}=\mathrm{O}$, and $-\mathrm{NH}_{2}$ groups. Their solubilities in aqueous solutions are higher than that of $1-\mathrm{CN}$ and 2-CT. In our previous study, PAB was found to be highly suitable for attracting compounds with moderate polarity, especially for aniline and phenol compounds. ${ }^{9,23}$ Therefore, all other compounds were prepared in $100 \mathrm{ppm}$ and absorbed using the PAB phase. The results for the absorption of $10 \mathrm{ppm}$ of $1-\mathrm{CN}$ and 2-CT by the PIB phase are shown in Fig. 3A. A $300-\mu \mathrm{L}$ portion of the prepared solutions was injected into a $10-\mathrm{cm}$ long hollow waveguide sampler in these experiments. The injected sample volume was approximately $300 \mu \mathrm{L}$ for a $10-\mathrm{cm}$ long sampler. As can be seen in this plot, the time required to reach equilibrium was extremely fast, within $5 \mathrm{~min}$. This short equilibrium time is perhaps caused by the large contact surface in the hollow waveguide. To further test the absorption time profile when the PAB phase was used, the remaining polar compounds were also injected at a volume of $300 \mu \mathrm{L}$. Typical absorption curves are shown in Fig. 3B. The samples for these experiments were prepared in $100 \mathrm{ppm}$ for absorption. As can be seen in this figure, the time to reach equilibrium was also very short, within 5 min. 

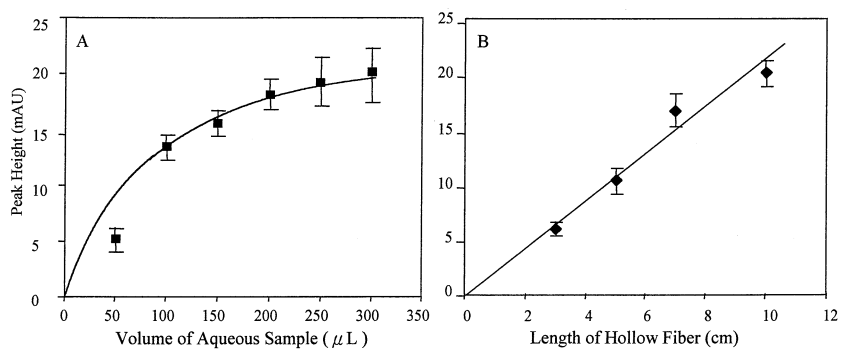

Fig. 5 (A) Effect of the sample volume on the analytical signals for 10-ppm 1-CN absorbed by PIB film. The data points were fitted with the curve shape in Eq. (2). Different amounts of aqueous samples were added into the hollow waveguide sampler following shaking of the sampler for 5 min. (B) Effect of the length of the sampler in the detection of a 10-ppm 1-CN solution. The sample volume was determined by filling the whole hollow waveguides.

\section{The effect of the SPME volume on the analytical signal}

According to Fig. 1B for the condition that sample volume is limited, the required $V_{\mathrm{f}}$ volume is smaller for analytes with a large $K$ than that with a small $K$. Moreover, to absorb 50\% of the analyte molecules in a sample volume of $V_{\mathrm{s}}$, the volume of the SPME phase should be equal to $V_{\mathrm{s}} / K$. To verify this behavior, 1-CN with 10-ppm was used. Different volumes of the SPME phase were obtained by coating waveguide samplers with different concentrations of PIB solution. The band located at $1230 \mathrm{~cm}^{-1}$ was used to indicate the relative amount of PIB coated inside the hollow waveguide sampler. To ensure that the equilibrium time did not greatly vary due to the thickness of the SPME phase, the absorption time profiles for different SPME phase thicknesses were taken first. The results are plotted in Fig. 4A. In this experiment, the PIB thickness was varied and its $1230 \mathrm{~cm}^{-1}$ band intensities were $0.714,1.175$ and $1.516 \mathrm{AU}$. As can be seen in this plot, the thickness of the SPME phase did not significantly impact the absorption time profile, and the equilibrium times to reach the maximum values were all around $5 \mathrm{~min}$. The results from absorbing $300 \mu \mathrm{L}$ of 10-ppm 1-CN using different SPME phase volumes for $20 \mathrm{~min}$ are shown in Fig. 4B. As can be seen in this plot, the relationship between the hydrophobic film volume and analytical signals fit with the curve shape in Eq. (2) very well, and the optimal signal could be obtained when the PIB band intensity reached close to 1.00.

\section{Effect of the sample volume on the analytical signals}

To study the effect of the sample volume, two types of experiments were performed. In the first type, the length of the hollow waveguide sampler was kept the same but the sample volume was varied. In this way, $V_{\mathrm{f}}$ remained constant, but $V_{\mathrm{s}}$ was changed. In the second type, the length of the hollow waveguide sampler was varied, and the aqueous sample filled up the hollow waveguide sampler. Accordingly, both $V_{\mathrm{f}}$ and $V_{\mathrm{s}}$ were changed. Also, a $10-\mathrm{cm}$ long PIB coated hollow waveguide sampler was first used to absorb different volumes of 10-ppm 1-CN in an aqueous solution. The volume of the sample was varied from $50 \mu \mathrm{L}$ to $300 \mu \mathrm{L}$. Sample volumes lower than $300 \mu \mathrm{L}$ could not fill the whole waveguide. Therefore, a gentle shaking of the waveguide sampler was performed to allow the aqueous solution to make complete contact with the hydrophobic film. According to the absorption time profiles in Fig. 3, 5-min of shaking was applied. The results are shown in Fig. 5A. As can be seen in this figure, the increase in the sample volume did affect the analytical signals, but after the sample volume reached $200 \mu \mathrm{L}$, the rate of increase

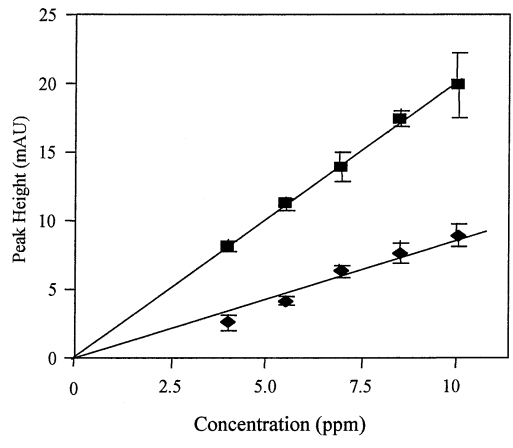

Fig. 6 (A) Different lengths of samplers were used to detect different concentration of $1-\mathrm{CN}$ in $10 \mathrm{ppm}$ concentration; $10-\mathrm{cm}$ and 3.5-cm long waveguide samplers were used for $300 \mu \mathrm{L}$ ( $)$ and 100 $\mu \mathrm{L}(\diamond)$ samples, respectively.

in the analytical signals decreased. The data points fitting the curve in Eq. (2) were highly matched with the theoretical curve, except for the data point for the $50 \mu \mathrm{L}$ sample.

In a second experiment, hollow waveguides were cut into 3,5 , 7, and $10 \mathrm{~cm}$ lengths. After filling with an aqueous sample containing $10 \mathrm{ppm}$ of $1-\mathrm{CN}$, the analytical signals were linearly related to the length of the hollow waveguide sampler. This indicated that as long as the ratio between $V_{\mathrm{f}}$ and $V_{\mathrm{s}}$ received constant, the percentage to absorb the molecules remained the same. Therefore, the smaller was the sample volume, the fewer was the number of absorbed molecules. Hence, the analytical signal was linearly related to the length of the hollow waveguide.

\section{Sample concentration effect on the analytical signals}

To examine the concentration effect, five concentrations of 1$\mathrm{CN}$ solutions were prepared: 4, 5.5, 7, 8.5 and $10 \mathrm{ppm}$. Two sample volumes were used, including 300 and $100 \mu \mathrm{L}$. The obtained signals are plotted against the $1-\mathrm{CN}$ concentrations in Fig. 6. As can be seen in this figure, the concentration did affect the analytical signals linearity in the way predicted in Eq. (4). To further study the concentration linearity with respect to the analytical signals, 1-CN and 2-CT solutions were prepared in the concentration range of 1 to $10 \mathrm{ppm}$ due to the low solubility in water. These samples were absorbed using PIB as the SPME phase. 4-NP, 4-CP, 2,3-DCP and ACe were prepared in the concentration range from 1 to $100 \mathrm{ppm}$. These samples were absorbed by the PAB phase. The sample volume was kept at $300 \mu \mathrm{L}$ with 10 -min absorption time. The obtained regression coefficients were $0.9953,0.9987,0.9993,0.9988,0.9988$, and 0.9996 for $1-\mathrm{CN}, 2-\mathrm{CT}, 4-\mathrm{NP}, 4-\mathrm{CP}, 2,3-\mathrm{DCP}$ and $\mathrm{AC}$, respectively. As the regression coefficients indicated, the linear relationship between the concentration and analytical signals were highly linearly related. Based on three-times the noise level in the spectra, the calculated detection limits were 0.59 , $1.21,0.79,0.81,1.77,1.63,0.25$, and $0.61 \mathrm{ppm}$ for $1-\mathrm{CN}, 2-\mathrm{CT}$, 4-NP, 4-CP, 2,3-DCP, AC, 3-NA, and 2,4-DCA, respectively.

\section{Stability of the hollow waveguide sampler in different $\mathrm{pH}$ solutions}

As in our previous analyses, PIB was highly stable in aqueous solutions at different $\mathrm{pH}$ values because of its low polarity nature. On the other hand, PAB contains a cyanide group that can interact with water when soaking in aqueous solutions. Some polar analytes are good proton acceptors or donors that can form charges in aqueous solutions and lower the 

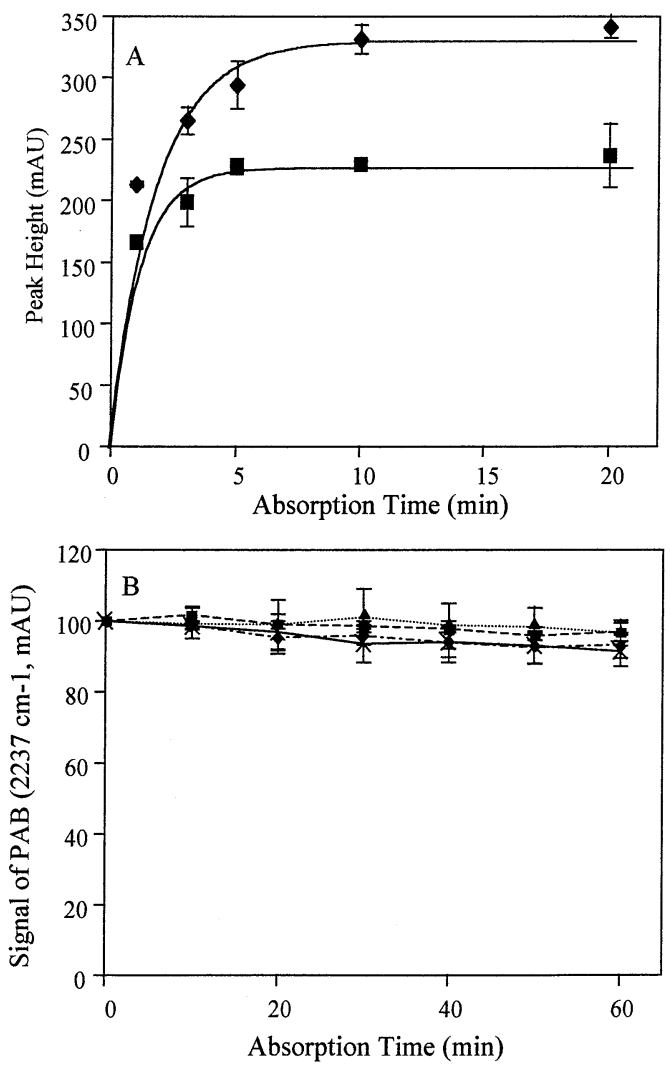

Fig. 7 (A) Absorption time profiles in determination of 100-ppm 2,4-DCA with ( $\bullet$ ) and without $\mathrm{pH}$ buffered ( $)$. A $0.1 \mathrm{M}$ sodium acetate solution was used as the buffer solution; its $\mathrm{pH}$ value was 7.56. (B) Stability of a hollow waveguide sampler in different buffer solutions. Four buffer solutions were used, including $0.1 \mathrm{M}$ of a solution of sodium acetate $(\boldsymbol{\Lambda})$, potassium sulfate $(\mathbf{-})$, potassium biphthalate $(\bullet)$, and sodium bicarbonate $(x)$. The forming $\mathrm{pH}$ values of the buffer solutions were 7.56, 10.49, 3.46, and 8.79 for sodium acetate, potassium sulfate, potassium biphthalate, and sodium bicarbonate solutions, respectively.

absorptivity of the polymer film to that particular analyte. Therefore, the stability of $\mathrm{PAB}$ was further examined in different $\mathrm{pH}$ solutions. The results are shown in Fig. 7. In this experiment, 2,4-DCA was used as the probe molecule and PAB was used to absorb this analyte. This compound can be protonated in acidic solutions, leading to low absorption by $\mathrm{PAB}$. After adjusting the $\mathrm{pH}$ value of the solution, higher signals were observed but the time to reach equilibrium was similar to that of unadjusted solutions. To extend the hollow waveguide sampler for examining compounds that can accept or donate protons, the hollow waveguide stability was examined in different buffer solutions, with the results shown in Fig. 7B. Very close $\mathrm{PAB}$ signals could be observed after soaking in buffer solutions for $60 \mathrm{~min}$. This indicated that the hollow waveguide sampler is applicable to aqueous samples with different $\mathrm{pH}$ values.

\section{Conclusion}

In this study, we successfully extended the IR hollow waveguide sensing method to detect organic compounds in sample volumes smaller than $300 \mu \mathrm{L}$. The detection limits using this method could be lower than $1 \mathrm{ppm}$ for the tested compounds. A theoretical consideration of the analytical working function for low volumes of samples was given in this study. To study the suitability of the hollow waveguide sampler in the detection of organic compounds in aqueous solutions, the factors that influenced the analytical signals were examined, including the effects of the volume, concentration and length of the hollow waveguide sampler. The results indicated that a large sample volume is not necessary, and even sample volumes less than $100 \mu \mathrm{L}$ can still be observed with a high-quality spectral result. The thickness of the hydrophobic film was very thin, leading to fast absorption of the analytes. In general, 5 min of equilibrium was enough time to reach the maximum absorption signals. The film thickness did affect the analytical signals, as theoretically predicted. Quantitative results could be easily obtained as long as all of the factors remained constant. The obtained linear regression coefficients in the standard curves were higher than 0.995 .

\section{Acknowledgements}

The authors would like to thank the National Science Council of the Republic of China for financially supporting of this work under Contract No. NSC90-2113-M-033-018.

\section{References}

1. N. J. Harrick, "Internal Reflection Spectroscopy", 1967, Wiley, New York.

2. A. Messica, A. Greestein, and A. Katzir, Appl. Optics, 1996, 35, 2274.

3. S. Simhony, A. Katzir, and E. M. Kosower, Anal. Chem., 1988, 60, 1908.

4. R. Gobel, R. Krska, R. Kellner, J. Kastner, A. Lambercht, M. Tacke, and A. Katzir, Appl. Spectrosc., 1995, 49, 1174.

5. P. H. Paul and G. Kychakoff, Appl. Phys. Lett., 1987, 51, 12.

6. K. Newby, W. M. Reichert, J. D. Andrade, and R. E. Benner, Appl. Opt., 1984, 23, 1812.

7. J. Yang and Y.-S. Huang, Appl. Spectrosc., 2000, 54, 202.

8. R. Krska, R. Kellner, U. Schiessel, M. Tacke, and A. Katzir, Appl. Phys. Lett., 1993, 63, 1868.

9. J. Yang and M.-L. Cheng, Analyst, 2001, 126, 881.

10. J. Yang and F.-P. Tsai, Anal. Sci., 2001, 17, 751.

11. J. Yang and J.-W. Her, Anal. Chem., 1999, 71, 1773.

12. J. Yang and J.-W. Her, Anal. Chem., 1999, 71, 4690.

13. J. Yang and H.-J. Lin, Analyst, 2000, 125, 1605.

14. J. Heo, M. Rodrigues, S. Saggese, and G. H. Sigel, Jr., Appl. Optics, 1991, 30, 3944.

15. M. C. Ertan-Lamontagne, S. R. Lowry, W. R. Seitz, and S. A. Tomellini, Appl. Spectrosc., 1995, 49, 1170.

16. R. Gobel, R. Krska, R. Kellner, R. W. Seitz, and S. A. Tomellini, Appl. Spectrosc., 1994, 48, 678.

17. D. S. Blair, L. W. Burgess, and A. M. Brodsky, Anal. Chem., 1997, 69, 2238.

18. Z. Zhang and J. Pawliszyn, Anal. Chem., 1993, 65, 1843.

19. Z. Zhang and J. Pawliszyn, J. High Resolut. Chromatogr., 1993, 16, 689.

20. Z. Zhang and J. Pawliszyn, Anal. Chem., 1995, 67, 34.

21. J. Yang, J.-W. Her, and S.-H. Chen, Anal. Chem., 1999, 71, 3740 .

22. J. Yang and J.-W. Her, Anal. Chem., 2000, 72, 878.

23. J. Yang and C.-P. Tsui, Anal. Chim. Acta, 2001, 442, 267.

24. R. P. Schwarzenbach, P. M. Gschwend, and D. M. Imboden, "Environmental Organic Chemistry", 1992, John Wiley \& Sons, Inc., New York. 